\title{
Charge Exchange in Atomic and Molecular Collisions
}

\author{
V. Sidis, Orsay \\ (Laboratoire des Collisions Atomiques et Moléculaires)
}

\section{Part I - Introduction and Experimental Methods}

Charge exchange in atomic and molecular physics is a collision process whereby one electron (at least) is transferred from one collision partner $(T)$ to the other $(P)$. Accordingly, the expression: charge exchange (CE), charge transfer, electron exchange, electron transfer, and electron capture may be used as synonyms.

$A C E$ reaction may be specified as:

$\mathrm{P}^{\alpha+}+\mathrm{T}^{\beta+} \rightarrow \mathrm{P}^{(\alpha-1)+}+\mathrm{T}^{(\beta+1)+}+\Delta \mathrm{E}$

where upper indices correspond to charge-states and $\Delta E$ is the energy gain $(>0)$ or loss $(<0)$ of the reaction. The process thus defined is thence seen to apply to singly $(\beta=1)$ or multiply $(\beta>1)$ charged positive ions colliding with neutrals, to ion-ion collisions $(\alpha, \beta>0)$, to ion-pair formation and mutual neutralisation $\left(\mathbf{P}+\mathbf{T} \rightarrow \mathbf{P}^{-}+\mathbf{T}^{+}\right)$as well as to electron transfer from negative ions $\left(P^{-}\right.$ $\left.+T-P+T^{-}\right)$. Likewise, the reaction may involve excited species e.g. : metastable reactant ions or neutrals, electronically excited products and, in the case when molecules are involved, ro- vibrationally excited species as well as fragmentation products.

\section{Practical Interest of Charge Exchange Phenomena}

CE has been a subject of great interest for about two decades owing to the crucial role played by this phenomenon in various areas of pure and applied physics. For example, CE has great importance in atmospheric and astrophysical studies e.g: formation and destruction of $\mathrm{H}^{+}, \mathrm{O}^{+}, \mathrm{N}^{+}, \mathrm{N}_{2}^{+}$ions in the upper atmosphere; cycles of formation of molecules or radicals $\mathrm{IOH}, \mathrm{CO}, \mathrm{H}_{2} \mathrm{O}$, $\mathrm{HCO}$ ) in interstellar clouds; destruction of multiply charged ions (of O, C, N, S, etc.) by $\mathrm{CE}$ with $\mathrm{H}$ and $\mathrm{He}$ near stellar and interstellar X-ray emitter media.

Another example is provided by controlled thermonuclear research based on tokamak devices. It seems that CE between injected $\mathrm{H}$ atoms and highly charged impurity ions of $\mathrm{W}, \mathrm{Mo}, \mathrm{Fe}$, or $\mathrm{M}, \mathrm{C}, \mathrm{O}$ could constitute a severe restriction on

Fig. 1 - a) Sketch of an apparatus devoted to growth and beam attenuation measurements (M. Matic et al., J. Phys. B 13 (1980) 3665. An ion-beam, extracted from a discharge ionsource, is accelerated to $2-30 \mathrm{keV}$ and momentum analysed by magnet $M$. The analyser $A$ removes possible Aston peaks (or neutrals) from the beam. The ion-beam is focussed into the interaction region IC which, for growth measurements, is filled with the reactant gas under low pressure; after the interaction the beam is charge analysed by condenser $C$ and the neutral component is detected by the secondary electron emission detector $F$. With IC empty, the beam is deflected towards the $127^{\circ}$ electrostatic analyser which serves, when filled with target gas, as a beam attenuation chamber. b) Sample CE cross-sections for $\mathrm{N}^{+}+$rare-gas collisions illustrating the variety of behaviours as a function of target, collision energy and excitation state of the projectile. Experimental data points have been obtained using the growth and attenuation methods (theoretical results have been determined from the universal curve $G$ of Fig. 14). 1: $N^{+}(3 P)+X e$ : $2: \mathrm{Kr} ; 3: \mathrm{Ar} ; 4: \mathrm{N}-\left({ }^{1} \mathrm{D}\right)+\mathrm{Kr} ; 5$ : $N+\left({ }^{5} S\right)+\mathrm{Ne} ; 6:$ He (Data from ref. quoted above).
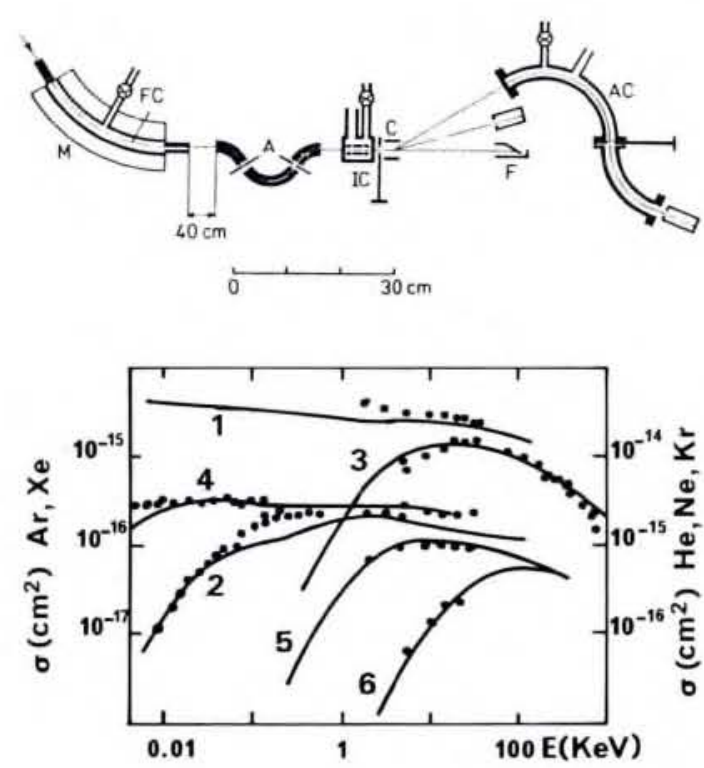

the effectiveness of the system: plasma cooling by radiative decay of the product ions having captured an electron in highly excited states, neutral $\mathrm{H}$ loss, production of impurities by collisions of the produced $\mathrm{H}^{+}$ions with the chamber walls, etc. More recently, research in inertial confinement by heavy ions has prompted the investigation of ion-ion CE in order to assess the limitations that might be caused by ion loss according to the reaction: $\mathrm{X}^{+}+\mathrm{X}^{+}-\mathrm{X}+\mathrm{X}^{2+}$.

CE is also of importance as a pumping mechanism in gas-phase lasers and work is currently under way to investigate its possible role as a pumping mechanism for plasma $X$-ray lasers. CE plays a central role in $\mathrm{H}^{-}$-beam and neutral-beam technologies for fusion plasma fuelling and heating and last, but not least, it constitutes a fundamental process in ion-chemistry.

A comprehensive understanding of CE phenomena and a detailed knowledge of their importance is hence an indispensable prerequisite for many research fields.

\section{Basic Research on Charge Exchange}

From a fundamental point of view, CE constitutes a special class of rearrangement processes exhibiting such a wealth of diversified features that it has intrigued physicists since its first identification by Henderson and Rutherford in the late 20's. Although the first attempts at a theoretical explanation of the phenomenon emerged in the 30's, the real outburst of systematic studies dates back to the 50's and the field has kept evolving steadily since then.

\section{Experimental Methods}

The set of experimental tools to study CE my be described by referring, except when stated otherwise, to the process: $\mathrm{P}^{+}+\mathrm{T} \rightarrow \mathrm{P}+\mathrm{T}^{+}$. When an ion beam $\left(\mathrm{P}^{+}\right\rangle$is passed through a gas cell or crossed with a neutral beam (T) one of the three following experimental methods to study CE may be used:

i) measurement of the intensity of neutralised projectiles $P$, 


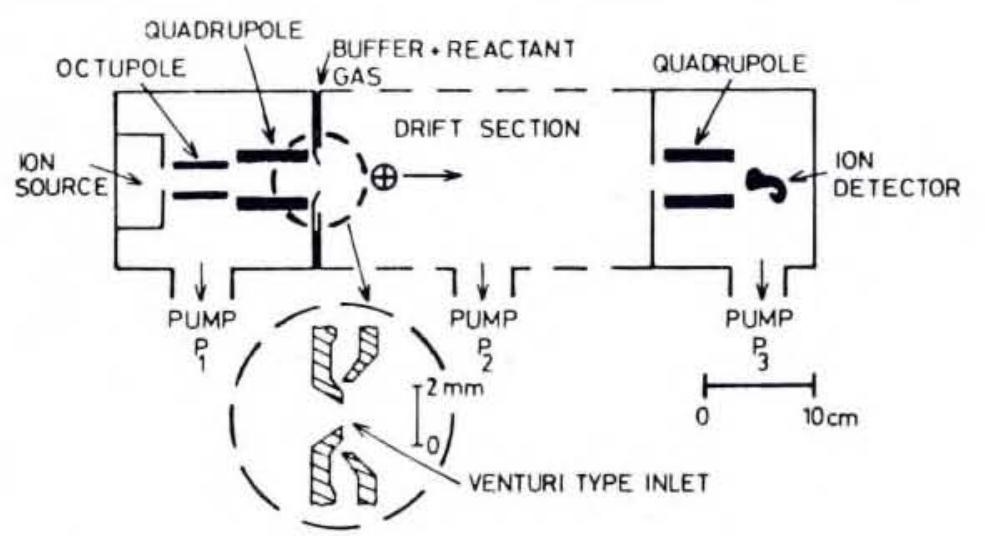

Fig. 2 - a) Sketch of a selected-ion drifttube apparatus (W. Lindinger in Electronic and Atomic Collisions, invited papers of XIII ICPEAC, Berlin, Eds. J. Eichler et al., North Holland, 1983). The source is an exchangeable low and high pressure electron impact and discharge ion-source. The octupole storage section allows the ions from the source to interact with neutrals to produce secondary ions in specific excited states. The quadrupole mass spectrometer preselects ions of one mass before introduction in the drift chamber.

b) Sketch of the CRESU apparatus (B. Rowe et al, J. Chem. Phys. 80 (1984) 241). Labels are as follows: BG buffer gas entry port; NR nozzle reservoir; N nozzle; EB electron beam that produces the ions; IC isentropic core; S skimmer; SW shock wave; QM quadrupole mass spectrometer. The various components are not necessarily to scale: the chamber diameter is $2 \mathrm{~m}$ and the typical diameter of the nozzle is $16 \mathrm{~cm}$.

ii) collection and analysis of the product ions $\mathrm{T}^{+}$.

iii) detection of characteristic emissions from the products, e.g.:

photons - when the products are formed in radiative states,

Auger electrons - when the products are formed in autoionising states (as in the case of electron capture from innershells),

dissociation fragments - as in the case of dissociative ion-molecule $\mathrm{CE}$

$$
\left(\mathrm{P}^{+}+\mathrm{AB} \rightarrow \mathrm{P}+\mathrm{A}+\mathrm{B}^{+}\right)
$$

When energetic $(E>100 \mathrm{eV}) \mathrm{P}^{\mathrm{a}+}$ beams are considered, methods of type (i) may be used. These are, on the one hand, the so-calied growth and beam attenuation experiments (Fig. 1a) that are performed at low target gas pressures to ensure single collision conditions which enables a direct measurement of the CE cross-section. Growth methods measure the emerging neutral intensity, or the intensity of particles having undergone a change of their charge-state, after having swept out the primary $\mathrm{P}^{\mathrm{a}+}$ ions by transverse electric or magnetic fields. Alternatively, attenuation methods measure the intensity of the attenuated primary beam of ions that is constrained, fields in the collision chamber itself, to follow a specific curved path (Fig. 1). On the other hand, experiments based on the so-called equilibrium

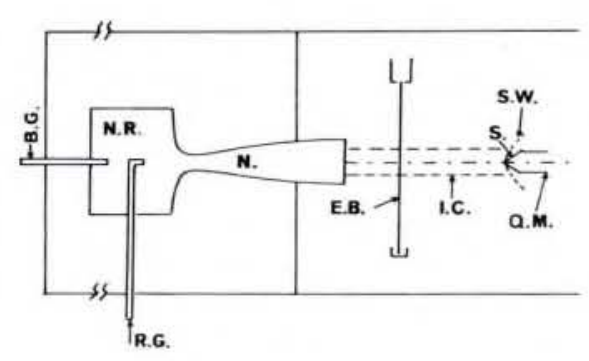

method are performed at sufficiently high pressures so that the fraction $f$ of the total beam leaving the cell in the charge state $j$ does not depend on pressure any more and is related to the corresponding CE cross-section $\sigma_{\text {, as: }}$ $f_{j}=\sigma_{j} / \Sigma$ where the denominator relates to all charge changing processes.

At low collision energies $(E<$ few eV), ground state neutrals may no longer be with the threshold electrons. detected satisfactorily by electron multipliers, therefore, most experiments are based either on an adaptation of the attenuation technique or on methods of type (ii) or both. One example is the selected ion drift tube technique (Fig. 2a). Here, mass selected ions in specific internal states of excitation enter a drift chamber filled with a buffer gas chosen for its inactivity (at pressures $<1$ Torr). The ions, subjected to a homogeneous electric field along the axis of the chamber, acquire a steady mean drift velocity parallel to the field. From the intensity of primary ions that reach the exit of the chamber one may deduce the reaction rate coefficient $k$, i.e. the cross-section averaged over the velocity distribution of the reactants.

A new method has been proposed to measure $C E$ reactions at energies prevailing in interstellar molecular clouds $(1-10 \mathrm{meV} \equiv 10-100 \mathrm{~K})$. This is the CRESU (cinétique de réactions en écoulement supersonique uniforme) technique (Fig. 2b) which makes use of a uniform supersonic jet, at pressures of $5 \times$ $10^{-3}-10^{-4}$ Torr, generated by Laval nozzles in a large rarefied wind tunnel. The reactant neutral gas $(T)$ is mixed with a buffer gas which is ionised by electron impact in the flow itself to produce the reactant ions $\left(\mathrm{P}^{+}\right)$. The linear decline of the reactant-ion flow rate at two different distances yields the reaction rate constant.

Another new method at low energies $(1 \mathrm{eV}<E<$ few tens of $\mathrm{eV})$ is the threshold electron-secondary-ion coincidence technique (Fig. 3a) that provides a nice example of both methods (i) and (ii).

Fig. 3 - a) Sketch of an apparatus based on the TESICO technique (Govers et al., Chem. Phys. 87 (1984) 373. Synchrotron radiation, from the ACO electron storage ring in Orsay, is focussed into the photoionisation region of the spectrometer. Photoelectrons are accelerated towards a detector and their time-of-flight is measured relative to the photon pulses. Only threshold electrons ( $w i t h$ energy less than $50 \mathrm{meV}$ ) are detected on the basis of angular and temporal discrimination. The electron signal triggers an electric pulse producing a 12.8 $\mathrm{V} / \mathrm{cm}$ electric field during $5 \mu \mathrm{s}$ that accelerates the ions towards an effusive jet of neutrals. Parent ions, of selected internal energy, and product ions are detected in delayed coincidence

b) Sensitivity of the total CE cross-section for the $\mathrm{N}_{2}^{+}+$Ar collision on the vibronic state of the molecular ion: left $\mathrm{N}_{2}^{+}\left(\mathrm{X}^{2} \Sigma^{+}, v\right)$, right $\mathrm{N}_{2}^{+}\left(\mathrm{A}^{2} \Pi, v\right)$. Results obtained using the threshold electron-secondary ion - coincidence technique (data from ref. quoted in Fig. 2a).
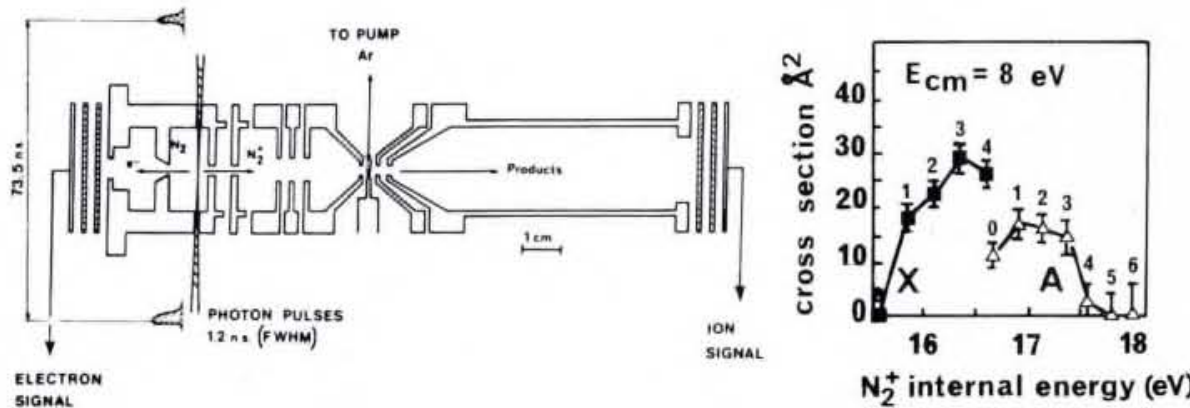

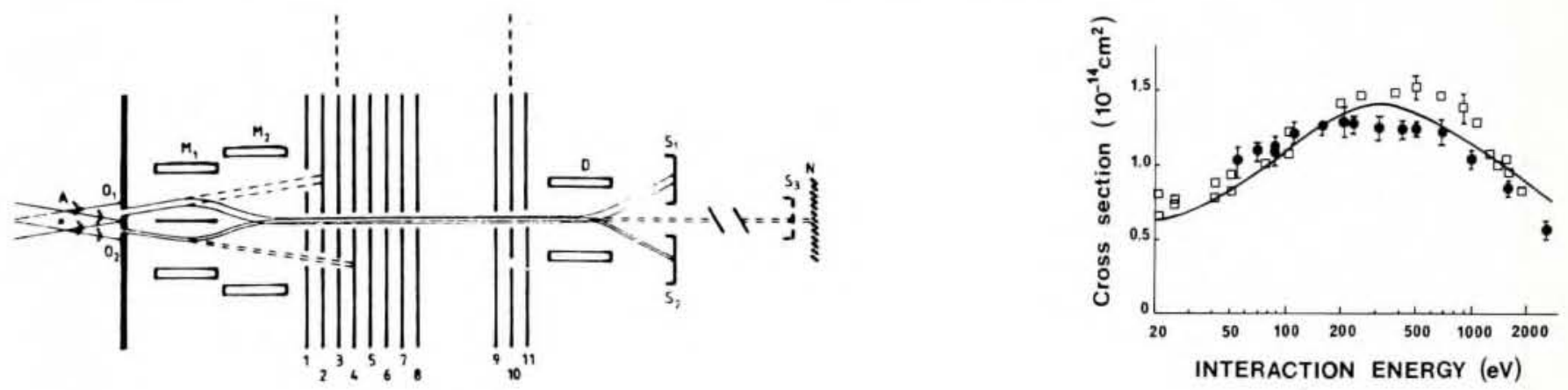

Fig. 4 - a) The merged-beams apparatus of Szucs et al. (J. Phys. B 17 (1984) 1613). $\mathrm{H}^{+}$and $\mathrm{H}^{-}$ion beams extracted from duoplasmatron sources and accelerated up to some $10 \mathrm{keV}$ are momentum analysed, focussed and directed so as to cross the axis of the apparatus at the same point $A$. The beams enter a high-vacuum chamber (10-10 Torr) through apertures $O$ and are then merged by means of two sets of deflecting plates $M$. The beams remain merged over a distance of $10 \mathrm{~cm}$. In this region a series of eleven thin metal plates enables one to adjust and control the relative energy of the beams and, in addition, to define precisely the interaction length. The beams are then demerged by deflector $\mathrm{D}$ and are collected in the Faraday cups $\mathrm{S}$ while neutrals are detected on the electron multiplier $\mathrm{N}$ located $128 \mathrm{~cm}$ behind the centre of the observation region. These signals are time analysed in order to extract the time correlated pairs produced by mutual neutralisation.

b) $\mathrm{H}^{+}+\mathrm{H}^{-}$mutual neutralisation cross-section obtained with the merged (open squares) and inclined (full circles) beam techniques (data from the reference quoted in Fig. 2a and from Peart et al., J. Phys. B 18 (1985) L439 resp.). The interest of these studies is that, triggered by theory (solid line, results of Sidis et al., Phys. Rev. Lett. 47 (1981) 1280) they have definitely disproven previous data which showed a huge cusp and additional structure in the energy range $30 \mathrm{eV}-200 \mathrm{eV}$.

Here, the reactant $\mathrm{P}^{+}$ions are prepared in controlled internal states by photoionisation at threshold wavelengths (using e.g. a synchrotron radiation facility). There ions are accelerated towards the reaction region where they collide with a neutral jet. The primary $\mathrm{P}^{-}$and secondary $\mathrm{T}^{+}$ions are recorded in delayed coincidence with the primary photoelectrons and the cross-sections are deduced from the relative importance of the $\mathrm{P}^{+}$and $\mathrm{T}^{+}$signals (Fig. 3).

Another quite powerful method to study almost all CE processes in the energy range $0.1 \mathrm{eV}<E<10^{4} \mathrm{keV}$ is the merged (Fig. 4a) or inclined beam technique. Here, the reactant particles $\mathrm{P}^{\alpha+}, \mathrm{T}^{\hat{\beta}+}$ are formed into high energy beams that are superposed or crossed at very small angles $\chi$. By varying $E_{\mathrm{PT}}$ and $\chi$ it is seen that a wide range of relative energies may be scanned. After the interaction region, the different charge components of the beam are separated and may be recorded coincidentally (Fig. 4). However, the great difficulty of mastering this technique (ultra-high vacuum $\cong 10^{-10}$ Torr, tenuous beams, problems related to the geometry of the interaction region, etc.) explains why, despite its broad potentialities, it is used only by a minority of experimental groups throughout the world.

All the afore-mentioned techniques aim at determining total charge changing cross-sections, but they provide little or no information on the internal states of the products nor do they lend themselves to a detailed understanding of the charge exchange mechanism as a function of the P-T distances of approach. Information on the internal states of the products may be obtained by indirect means, or by methods of type (iii) or by laser induced fluorescence probing of the products. The latter method is being increasingly used to determine the rotational and vibrational populations of the

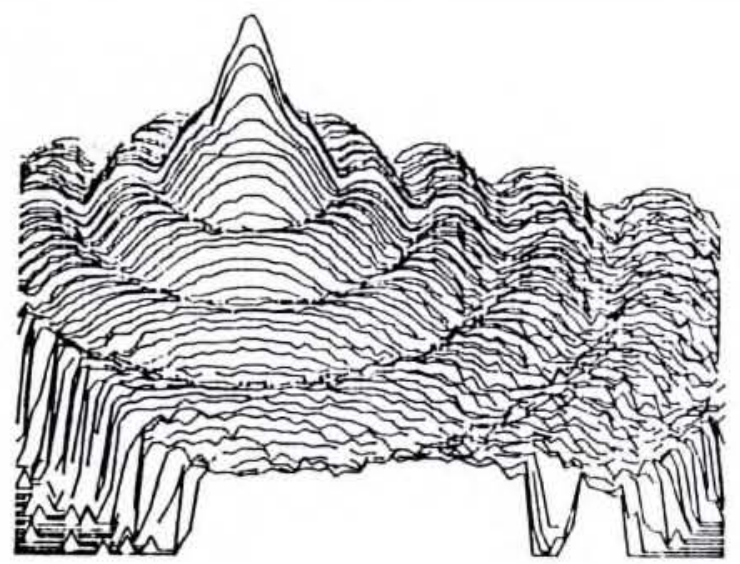

Fig. 5 -- Logarithmic intensity, as a function of position on a microchannel plate detector, of product neutrals from the $\mathrm{He}^{-}$ + He collision at $750 \mathrm{eV}$ (lab.) (data from J.H. Newman et al. XIII ICPEAC, Berlin 1983, p. 480 ). The observed structures illustrate the interference pattern discussed in the theoretical section in connexion with resonant $C E$. product in ion + molecule reactions. It consists of scanning an appropriate tunable laser through absorption bands of the molecular product and recording the subsequent fluorescence intensities that reflect, under specific operational conditions, the state distribution of the product sought.

Owning to the existence of (semiclassical) relations between the distance of closest approach and the scattering angle, information on the dependence of CE upon characteristic P-T distances may be obtained from experiments that determine angular differential-cross sections. One possibility consists of measuring the intensity of scattered neutrals, irrespective of their internal state and that of the product ion, as a function of scattering angle using a movable detector or, better, by performing simultaneous measurements at all angles using a position-sensitive device based on microchannel plate detectors (Fig. 5). Additional information on the internal states of the products may be obtained my measuring the energy losses or gains undergone by the scattered particles at each angle. This is the basic principle on which rests so-called collision spectroscopy. As an example, CE excitation processes involving ions in low charge-states: $\mathrm{P}^{2+}+\mathrm{T} \rightarrow \mathrm{P}^{(*)}+\mathrm{T}^{+}$ processes became possible only with the advent of time of flight techniques, in the early 70's (Fig. 6). The best resolution $(\cong 0.1 \mathrm{eV})$ are attained with the longest flight distances $(3-7 \mathrm{~m})$ and the lowest collision energies (few tens of $\mathrm{eV}$ ) compatible with a decent detection of neutrals. 

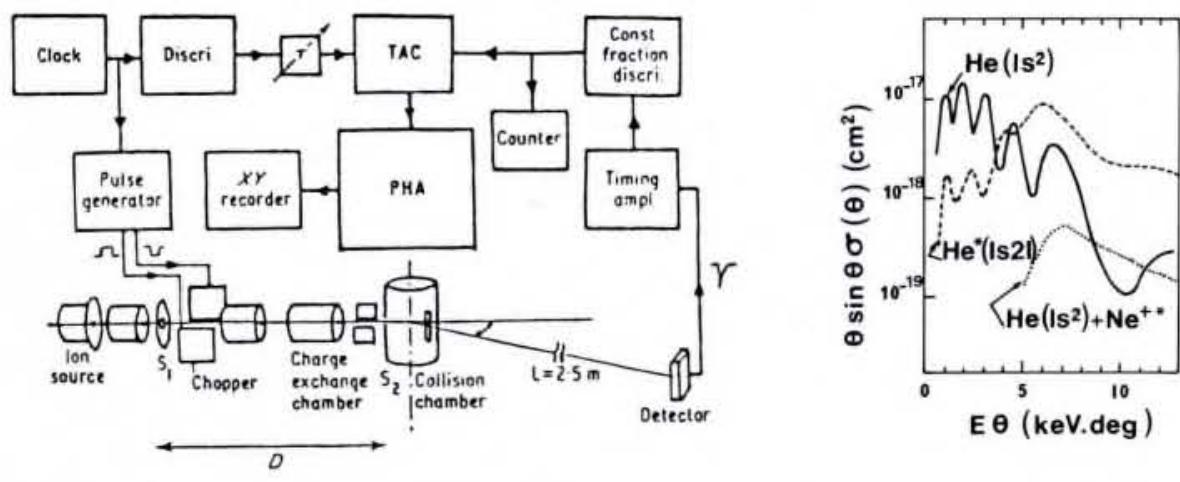

Fig. 6 - a) Sketch of a time-of-flight spectrometer devoted to the measurements of doubly differential (i.e. in energy and scattering angle) cross-sections (J.C. Brenot et al., J. Phys. B 8 (1975) 448). An ion beam extracted from a discharge ion source is focussed and then chopped as it passes through a parallel-plate capacitor delivering a square wave pulse $( \pm 10 \mathrm{~V})$ whose frequency is adjustable between 0.1 and $1 \mathrm{MHz}$. (The charge exchange cell in front of the collision chamber produces neutrals for $P+T$ collision studies.) For the purpose of analysing the scattered neutrals, ions are swept out by an electric field at the exit of the collision chamber. A planar detector - a microchannel plate — may be placed at different flight distances $L \leq 7.5 \mathrm{~m}$ from the collision chamber. The time spent by the scattered neutrals to reach the detector is measured by a standard technique involving a time to amplitude converter and a pulse height analyser; the calibration of the energy loss scale is obtained using a delay line. The section from the source to the collision chamber is actually the rotatable part of the apparatus $\left(-5^{\circ} \leq \Theta \leq 15^{\circ}\right)$.

b) Reduced doubly differential cross sections for $\mathrm{CE}$ in the $\mathrm{He}^{+}+\mathrm{Ne}$ collision at $1500 \mathrm{eV}$ (lab.) (data from Barat et al., J. Phys. B 9 (1976) 269). CE into $\mathrm{He}\left(1 \mathrm{~s}^{2}\right)$, full line, illustrates the behaviour as a function of scattering angle of the near resonant $C E$ process discussed in the theoretical section. The CE-excitation processes are the result of a combination of electron transition mechanisms at small ion-atom separation and sharing mechanisms at large separations (see theoretical section).
Measurements on charge exchange between highly charged ions and neutrals have awaited the late 70's owing primarily to the unavailability until then of intense sources of highly charged ions. Today, such ions are almost routinely produced by electron cyclotron resonance ion sources or electron beam ion-sources thereby explaining the recent tremendous increase of the experimental literature on $\mathrm{CE}$ involving highly charged ions. Again, the first experiments dealt with total cross-sections and change-state dependences.

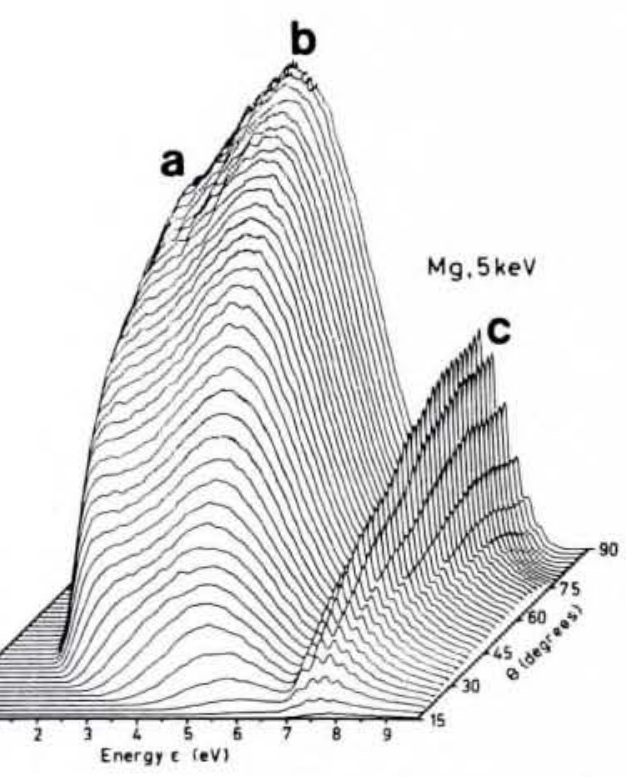

Fig. 8 - Time and position coincidence spectrum of $\mathrm{H}$ fragments from dissociative $\mathrm{CE}$ in the $\mathrm{H}_{2}^{+}+\mathrm{Mg}$ collision at $5 \mathrm{keV}$ (lab.) (data from de Bruijn et al. (Chem. Phys. 85 (1984) 215). The shoulder at small dissociation energies is attributed to $\mathrm{CE}$ into the $\mathrm{a}^{3} \Sigma_{\mathrm{g}}^{-}$state of $\mathrm{H}_{2}$ that radiates to the $b^{3} \Sigma_{u}^{+}$dissociative state, the main structure around $4-5 \mathrm{eV}$ is due to $\mathrm{CE}$ into the $\mathrm{b}$-state itself and the peaks above $7 \mathrm{eV}$ are due to $C E$ into the predissociative $\mathrm{c}^{3} \Pi_{u}$ state. The 2-dimensional plot illustrates the dependence of dissociative CE upon orientation of the impinging molecular-ion.

Fig. 7 - a) Sketch of the spectrometer used by P. Roncin et al. (J. Phys. $E$, in press) to measure doubly differential CE cross-sections for collisions between multiply-charged ions and neutrals. Multiply-charged ions $\mathrm{P}^{a+}$ extracted from the ECR ion-source in Grenoble undergo $C E$ in a collision chamber. Product ions $\mathrm{P}^{\mathrm{a}-1}$ (or $\mathrm{Pa}^{\mathrm{a}-2}$ ) enter a parallel plate electrostatic analyser under $45^{\circ}$ inclination. After deflection by the plates of the analyser they hit a two-dimensional position sensitive detector. Parametric relations between the position on the detector, the energy of the particles and their angle of incidence on the analyser enable one to deduce the angular differential cross-section for each characteristic energy gain of the CE reaction.

b) Dependence as a function of the laboratory scattering angle of an energy gain spectrum for $\mathrm{N} 6+^{*}$ (n) ions scattered from the $\mathrm{N}^{7+}+\mathrm{H}_{2}$ collision at $E_{\text {lab }}=28 \mathrm{keV}$. The CE process is seen to populate selectively the $n=4$ and 5 levels in forward scattering: data from Roncin et al. to be published. (The weak bump to the left of the main peaks should be ignored.)

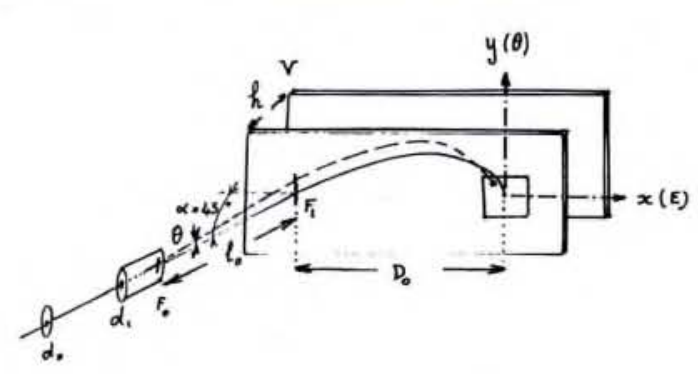

Information on the specific state population arose subsequently from the measurement of the light emitted from the excited product ions. Only recently have doubly differential cross-section measurements been reported. In the most recent experiment of this type (Fig. 7a), the energy of the scattered $\mathrm{P}^{(\alpha-1)+}$ particles is analysed using a parallel plate electrostatic analyser and the differential cross-sections are deduced from a two-dimensional position-detection of the particles (Fig. 7)

To end this section on experimental methods, let us also mention doubly differential photon-neutral coincidence experiments on single and double electron capture from K-shells at a few MeV/amu, detailed studies of dissociative $\mathrm{CE}$ in molecular-ion + atom collisions using a time- and position-detection of both neutral molecular fragments (Fig. 8), doubly differential crossed beam experiments at energies as low as $0.5 \mathrm{eV}$... the list could go on.

Part II on the theoretical aspects will be published in the June issue of $E N$. 